\title{
Affinity Capture of p97 with Small Molecule Ligand Bait Reveals a 3.6 Ångstrom Double-hexamer Cryo-Electron Microscopy Structure
}

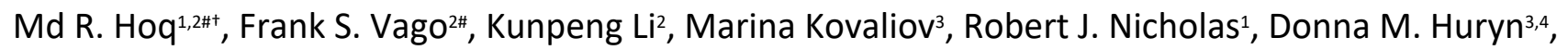
Peter Wipf ${ }^{5}$, Wen Jiang ${ }^{1,2,6 *}$ and David H. Thompson ${ }^{1,6 *}$

\section{Affiliations}

${ }_{1}^{1}$ Department of Chemistry, Purdue University, West Lafayette, Indiana 47907, 2 Department of Biological Sciences, Purdue University, West Lafayette, Indiana 47907, ${ }^{3}$ University of Pittsburgh Chemical Diversity Center, University of Pittsburgh, Pittsburgh Pennsylvania 15261, “ Department of Pharmaceutical Sciences, School of Pharmacy, University of Pittsburgh, Pittsburgh, Pennsylvania 15261. ${ }^{5}$ Department of Chemistry, University of Pittsburgh, Pittsburgh, Pennsylvania 15260. ${ }^{6}$ Purdue Center for Cancer Research, West Lafayette, Indiana 47907

\section{Supplementary Materials and Methods}

Scheme S1. Synthesis of Compounds $1-11$ and DBCO-PEG5000-DSPE

p97 Purification Protocol

\section{Supplementary Figures}

Figure S1. ${ }^{1} \mathrm{H}-\mathrm{NMR}$ of DBCO-PEG5000-DSPE

Figure S2. ${ }^{1} \mathrm{H}-\mathrm{NMR}$ of $\mathbf{1}$ (Cyanoindole ligand-PEG5000-DSPE conjugate)

Figure S3. ${ }^{1} \mathrm{H}-\mathrm{NMR}$ of 3 (3-Tolyl triazole-PEG5000-DSPE conjugate)

Figure S4. ${ }^{1} \mathrm{H}-\mathrm{NMR}$ of 4 (Indole ligand-PEG5000-DSPE conjugate)

Figure S5. SEC Chromatogram

Figure S6. DLS of SEC Fractions

Figure S7. Negative stain TEM of p97 captured by 1:99 1:mPEG750-DSPE coated grids using different concentrations of $\mathrm{p} 97$

Figure S8. Negative-stain and cryo-EM images of p97 captured with grids coated with different 1:mPEG750-DSPE ratios.

Figure S9. Quality of acquired data

Figure S10. Comparison of our cryo-EM maps vs. the reference p97 map 

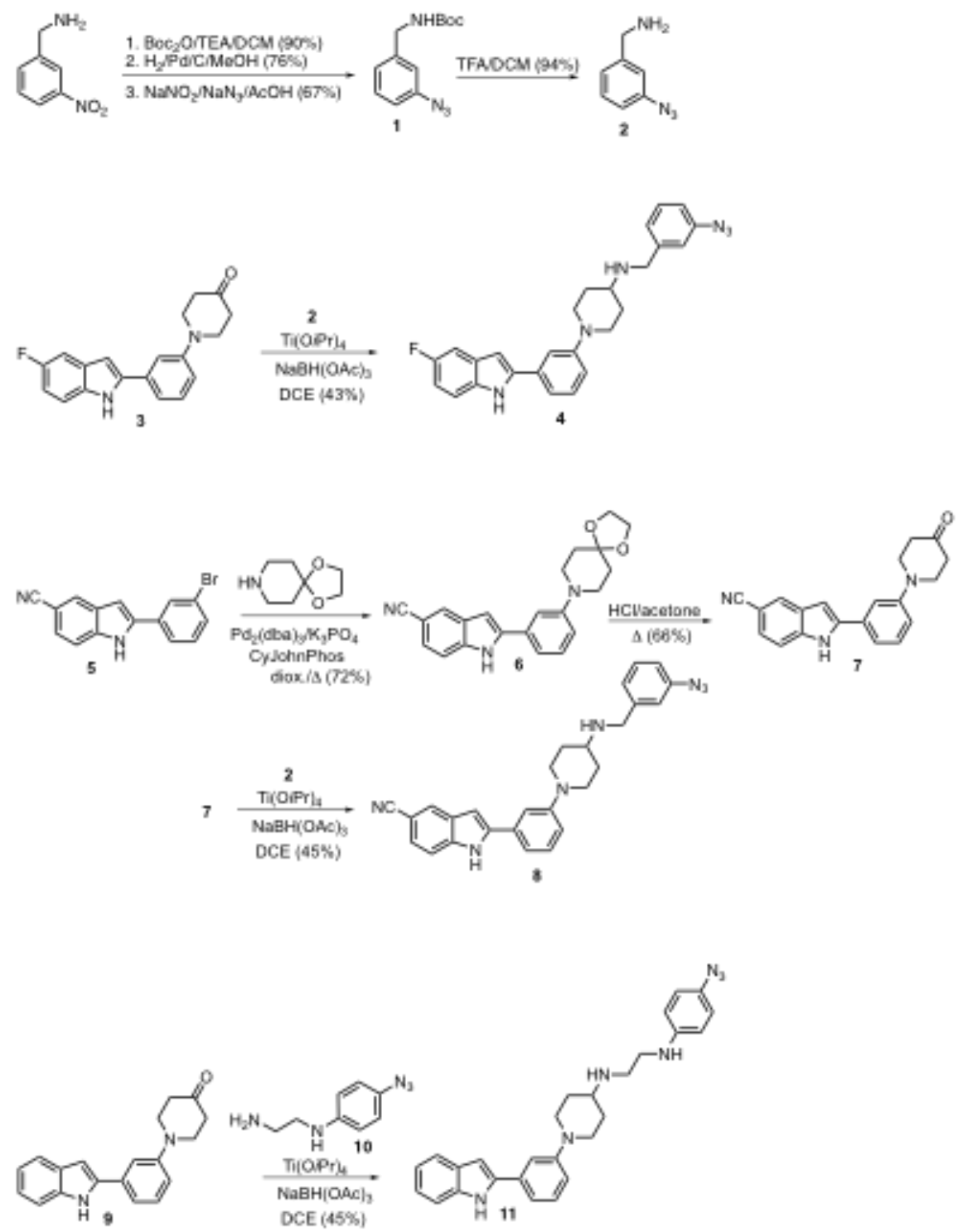

Scheme 1. Synthesis of azido analogs.

tert-Butyl (3-nitrobenzyl)carbamate. A procedure adapted from CLM Goodyer et al. (Bioorg. Med. Chem. 11, 4189-4206 (2003)) was used. To a solution of $\mathrm{Boc}_{2} \mathrm{O}$ (1.59 g, $7.23 \mathrm{mmol}$ ) in $\mathrm{CH}_{2} \mathrm{Cl}_{2}(10 \mathrm{~mL})$ was added a suspension of 3-nitrobenzylamine $(1.00 \mathrm{~g}, 6.57 \mathrm{mmol})$ and $\mathrm{Et}_{3} \mathrm{~N}$ $(0.73 \mathrm{~g}, 7.23 \mathrm{mmol})$ in $\mathrm{CH}_{2} \mathrm{Cl}_{2}(10 \mathrm{~mL})$ for $5 \mathrm{~min}$ at $0{ }^{\circ} \mathrm{C}$. The reaction was stirred at room temperature overnight. The reaction mixture was treated with $\mathrm{H}_{2} \mathrm{O}(10 \mathrm{~mL})$ and extracted with $\mathrm{CH}_{2} \mathrm{Cl}_{2}(3 \mathrm{x})$. The combined organic layer was washed with $\mathrm{H}_{2} \mathrm{O}$, brine, dried $\left(\mathrm{Na}_{2} \mathrm{SO}_{4}\right)$, filtered and concentrated to give product as a white foamy solid (1.5 g, 90\%). ${ }^{1} \mathrm{H}$ NMR $\left(500 \mathrm{MHz}, \mathrm{CDCl}_{3}\right) \delta 8.15-8.12(\mathrm{~m}, 2 \mathrm{H}), 7.63(\mathrm{~d}$, $\mathrm{J}=7.6 \mathrm{~Hz}, 1 \mathrm{H}), 7.51(\mathrm{t}, \mathrm{J}=7.9 \mathrm{~Hz}, 1 \mathrm{H}), 5.02(\mathrm{~s}, 1 \mathrm{H}), 4.42(\mathrm{~d}, \mathrm{~J}=5.0 \mathrm{~Hz}, 2 \mathrm{H}), 1.47(\mathrm{~s}, 9 \mathrm{H}) ; \mathrm{HRMS}(\mathrm{ESI}) \mathrm{m} / \mathrm{z}$ calcd. for $\mathrm{C}_{8} \mathrm{H}_{9} \mathrm{O}_{4} \mathrm{~N}_{2}(\mathrm{M}+\mathrm{H}-\mathrm{tBu})$ 197.0557, found 197.0557. 
tert-Butyl (3-aminobenzyl)carbamate. A procedure adapted from YR Choi et al. (Chem. Commun. 50, 15305-15308 (2014)) was used. A solution of tert-butyl (3-nitrobenzyl)carbamate $(1.50 \mathrm{~g}, 5.95 \mathrm{mmol})$ in $\mathrm{MeOH}(20 \mathrm{~mL})$ was evacuated and flushed with $\operatorname{Ar}(3 \mathrm{x})$ and treated with $10 \% \mathrm{Pd} / \mathrm{C}(0.06 \mathrm{~g}, 5.95 \mathrm{mmol})$. The reaction mixture was evacuated and subjected to $\mathrm{H}_{2}$ ( 1 atm balloon). After $18 \mathrm{~h}$, the reaction mixture was filtered through Celite ${ }^{\circledR}$, rinsed with $\mathrm{MeOH}$ and concentrated to give product $(1.00 \mathrm{~g}, 76 \%)$ as a brown oil that was used without further purification. ${ }^{1} \mathrm{H} \mathrm{NMR}(500 \mathrm{MHz}$, $\mathrm{CDCl} 3) \delta 7.11$ (td, J= 7.7, 2.9 Hz, $1 \mathrm{H}), 6.69-6.58(\mathrm{~m}, 3 \mathrm{H}), 4.80-4.79(\mathrm{~m}, 1 \mathrm{H}), 4.22(\mathrm{~d}, \mathrm{~J}=5.2 \mathrm{~Hz}, 2 \mathrm{H}), 1.46$ (s, $9 \mathrm{H})$; HRMS (ESI) m/z calcd. for $\mathrm{C}_{8} \mathrm{H}_{11} \mathrm{O}_{2} \mathrm{~N}_{2}(\mathrm{M}+\mathrm{H}-\mathrm{tBu})$ 167.0815, found 167.0813 .

tert-Butyl (3-azidobenzyl)carbamate (1). A solution of $\mathrm{NaNO}_{2}(0.45 \mathrm{~g}, 6.52 \mathrm{mmol})$ in water $(10 \mathrm{~mL})$ was added dropwise to an ice-cooled solution of tert-butyl (3-aminobenzyl)carbamate $(1 \mathrm{~g}, 4.50 \mathrm{mmol})$ in $20 \mathrm{~mL}$ of $\mathrm{AcOH}$. The reaction mixture was stirred $30 \mathrm{~min}$ at $0{ }^{\circ} \mathrm{C}$ and $\mathrm{NaN}_{3}$ $(0.22 \mathrm{~g}, 3.32 \mathrm{mmol})$ in $5 \mathrm{~mL}$ of $\mathrm{H}_{2} \mathrm{O}$ was then added dropwise in the dark. After $1 \mathrm{~h}$, the reaction

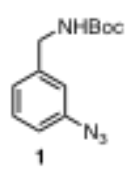
mixture was extracted with EtOAc and washed with satd. $\mathrm{NaHCO}_{3}(4 \mathrm{x})$. The organic layer was washed with water, brine, dried $\left(\mathrm{MgSO}_{4}\right)$, filtered and concentrated to give product as a colorless oil $(\mathbf{1}, 0.75 \mathrm{~g}$, $67 \%$ ) that was used without further purification. IR (neat) 3340, 2972, 2928, 2106, 1677, 1517, 1274, 1155, $797 \mathrm{~cm}-1 ;{ }^{1} \mathrm{H}$ NMR $\left(400 \mathrm{MHz}, \mathrm{CDCl}_{3}\right) \delta 7.31$ (td, J= 7.6, 0.9 Hz, $\left.1 \mathrm{H}\right)$, 7.06-7.04 (m, $\left.1 \mathrm{H}\right), 6.94-6.91$ $(\mathrm{m}, 2 \mathrm{H}), 4.31-4.30(\mathrm{~m}, 2 \mathrm{H}), 1.46(\mathrm{~s}, 9 \mathrm{H}) ; \mathrm{HRMS}(\mathrm{ESI}) \mathrm{m} / \mathrm{z}$ calcd. for $\mathrm{C}_{8} \mathrm{H}_{9} \mathrm{O}_{2} \mathrm{~N}_{4}(\mathrm{M}+\mathrm{H}-\mathrm{tBu})$ 193.0720, found 193.0719.

(3-Azidophenyl)methanamine (2). A procedure adapted from LL Alajarín \& J Berná (Tetrahedron 62, 6190-6202 (2006)) was used. To a solution of tert-butyl (3-azidobenzyl)carbamate (1, $0.75 \mathrm{~g}, 3.02)$ in $\mathrm{CH}_{2} \mathrm{Cl}_{2}(10 \mathrm{~mL})$ was added TFA dropwise at $0{ }^{\circ} \mathrm{C}$. The solution was warmed to room temperature. After $1 \mathrm{~h}$, the solution was concentrated, and the residue was diluted with

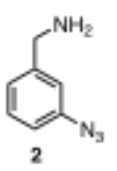
satd. $\mathrm{NaHCO}_{3}$ and extracted with EtOAc (3x). The combined organic layer was washed with brine, dried $\left(\mathrm{Na}_{2} \mathrm{SO}_{4}\right)$, filtered and concentrated to give product as an oil $(2,0.42,94 \%)$ that was used without further purification. ${ }^{1} \mathrm{H} N M R\left(500 \mathrm{MHz}, \mathrm{CDCl}_{3}\right) \delta 7.31(\mathrm{t}, \mathrm{J}=7.8 \mathrm{~Hz}, 1 \mathrm{H}), 7.09(\mathrm{~d}, \mathrm{~J}=7.6 \mathrm{~Hz}, 1 \mathrm{H}), 7.00(\mathrm{~s}, 1 \mathrm{H}), 6.92-$ $6.90(\mathrm{~m}, 1 \mathrm{H}), 3.87$ (s, $2 \mathrm{H}), 1.97$ (bs, $2 \mathrm{H})$; HRMS (ESI) m/z calcd. for $\mathrm{C}_{7} \mathrm{H}_{9} \mathrm{~N}_{4}(\mathrm{M}+\mathrm{H})$ 149.0822, found 149.0822.

2-(3-(1,4-Dioxa-8-azaspiro[4.5]decan-8-yl)phenyl)-1H-indole-5-carbonitrile (6). A solution of 2-(3-bromophenyl)-1H-indole-5-carbonitrile (5, $0.150 \mathrm{~g}, 0.505 \mathrm{mmol}$; JC Burnett et al. Org. Biomol. Chem. 15, 4096-4114 (2017)), $\mathrm{K}_{3} \mathrm{PO}_{4}(0.161 \mathrm{~g}, 0.757 \mathrm{mmol})$ was treated with dioxane $(1 \mathrm{~mL}$, degassed) followed by CyJohnPhos $(0.014 \mathrm{~g}, 0.040 \mathrm{mmol})$, $\mathrm{Pd}_{2} \mathrm{dba}_{3}(0.009 \mathrm{~g}, 0.010 \mathrm{mmol})$ and 1,4-dioxa-8-azaspiro[4.5]decane $(0.087 \mathrm{~g}, 0.607$

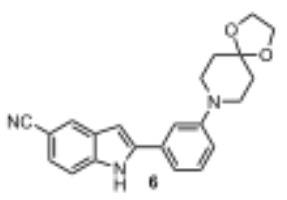
$\mathrm{mmol}$ ). The solution was degassed for $10 \mathrm{~min}$ and the reaction vial was sealed and submerged into preheated oil bath at $120^{\circ} \mathrm{C}$ and kept at this temperature overnight. The solution was cooled to room temperature and extracted with EtOAc, washed with brine, dried $\left(\mathrm{Na}_{2} \mathrm{SO}_{4}\right)$, filtered and concentrated. The crude residue was purified by chromatography on $\mathrm{SiO}_{2}\left(0-3 \% \mathrm{MeOH} / \mathrm{CH}_{2} \mathrm{Cl}_{2}\right)$ to give the product as an orange foam $(6,0.130 \mathrm{~g}, 72 \%) .{ }^{1} \mathrm{H}$ NMR $\left(500 \mathrm{MHz}\right.$, acetone- $\left.\mathrm{d}_{6}\right) \delta 11.20(\mathrm{~s}, 1 \mathrm{H}), 8.02-7.99(\mathrm{~m}, 1 \mathrm{H}), 7.57-$ $7.55(\mathrm{~m}, 1 \mathrm{H}), 7.51-7.48(\mathrm{~m}, 1 \mathrm{H}), 7.40(\mathrm{dd}, \mathrm{J}=8.4,1.5 \mathrm{~Hz}, 1 \mathrm{H}), 7.34-7.29(\mathrm{~m}, 2 \mathrm{H}), 7.04(\operatorname{app~d}, \mathrm{J}=0.7 \mathrm{~Hz}$, $1 \mathrm{H}), 7.01(\mathrm{dt}, \mathrm{J}=7.2,2.1 \mathrm{~Hz}, 1 \mathrm{H}), 3.97(\mathrm{~s}, 4 \mathrm{H}), 3.42(\mathrm{t}, \mathrm{J}=5.7 \mathrm{~Hz}, 4 \mathrm{H}), 1.80(\mathrm{t}, \mathrm{J}=5.8 \mathrm{~Hz}, 4 \mathrm{H})$; HRMS (ESI) $\mathrm{m} / \mathrm{z}$ calcd. for $\mathrm{C}_{22} \mathrm{H}_{22} \mathrm{O}_{2} \mathrm{~N}_{3}(\mathrm{M}+\mathrm{H}) 360.1707$, found 360.1705 . 
2-(3-(4-Oxopiperidin-1-yl)phenyl)-1H-indole-5-carbonitrile (7). To a solution of 2-(3(1,4-dioxa-8-azaspiro[4.5]decan-8-yl)phenyl)-1H-indole-5-carbonitrile $(6,0.26 \mathrm{~g}, 0.72$ $\mathrm{mmol})$ in acetone $(90 \mathrm{~mL})$ was added $3 \mathrm{M} \mathrm{HCl}(90 \mathrm{~mL})$ at room temperature. The solution was heated to reflux. After $2.5 \mathrm{~h}$, the solution was cooled to $0{ }^{\circ} \mathrm{C}$ and slowly neutralized with $\mathrm{Na}_{2} \mathrm{CO}_{3}$. The mixture was extracted with EtOAc, washed with brine, dried

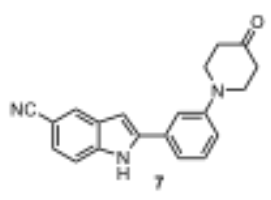
$\left(\mathrm{Na}_{2} \mathrm{SO}_{4}\right)$, filtered and concentrated. The residue was purified by chromatography on $\mathrm{SiO}_{2}(20-33 \%$ EtOAc/hexanes) to give the product as a yellowish-orange solid $(7,0.15 \mathrm{~g}, 66 \%) .{ }^{1} \mathrm{H} \mathrm{NMR}(400 \mathrm{MHz}$, $\left.\mathrm{CDCl}_{3}\right) \delta 8.89(\mathrm{~s}, 1 \mathrm{H}), 7.96(\mathrm{~d}, \mathrm{~J}=0.7 \mathrm{~Hz}, 1 \mathrm{H}), 7.47-7.38(\mathrm{~m}, 3 \mathrm{H})$, 7.27-7.26 (m, $\left.2 \mathrm{H}\right)$, 7.19-7.17 $(\mathrm{m}, 1 \mathrm{H})$, 7.01-6.98 (m, $1 \mathrm{H}), 6.86(\mathrm{t}, \mathrm{J}=1.0 \mathrm{~Hz}, 1 \mathrm{H}), 3.69(\mathrm{t}, \mathrm{J}=6.1 \mathrm{~Hz}, 4 \mathrm{H}), 2.61(\mathrm{t}, \mathrm{J}=6.1 \mathrm{~Hz}, 4 \mathrm{H}) ; \mathrm{HRMS}(\mathrm{ESI}) \mathrm{m} / \mathrm{z}$ calcd. for $\mathrm{C}_{20} \mathrm{H}_{18} \mathrm{O}_{2} \mathrm{~N}_{3}(\mathrm{M}+\mathrm{H}) 316.1442$, found 316.1442 .

2-(3-(4-((3-Azidobenzyl)amino)piperidin-1-yl)phenyl)-1H-indole-5-carbonitrile adapted from Huryn, D.M.; Wipf, P.; LaPorte, M.G.; Colombo, R.; Kovaliov, M.; Lim, C.; Alverez, C.N.; Yue, Z.; Samankumara, L.P.; Chatterley, A.J.; Yan, Y.; Liang, M.; Green, N.J.; Moore, W.; Baldwin, E.; Arkin, M.R.; Neitz, J.; Bulfer, S.; Ang, K.H.; Clifford, B. "Phenylindole derivatives as allosteric inhibitors of p97 ATPase and their preparation", WO2017/070320 was used. A solution of 2-(3-(4-oxopiperidin-1-yl)phenyl)-1H-indole-5-carbonitrile $(7,0.070 \mathrm{~g}, 0.221 \mathrm{mmol})$ in 1,2-

(8). A procedure DCE (4 mL) was treated with (3-azidophenyl)methanamine $(2,0.049 \mathrm{~g}, 0.332 \mathrm{mmol})$ followed by $\mathrm{Ti}(\mathrm{OiPr})_{4}$ $(0.074 \mathrm{~mL}, 0.244 \mathrm{mmol})$ at room temperature. After $30 \mathrm{~min}, \mathrm{NaBH}(\mathrm{OAc})_{3}(0.017 \mathrm{~g}, 0.082 \mathrm{mmol})$ was added. After $2.5 \mathrm{~h}$, additional $\mathrm{NaBH}(\mathrm{OAc})_{3}(0.017 \mathrm{~g}, 0.082 \mathrm{mmol})$ was added. Additional $\mathrm{NaBH}(\mathrm{OAc})_{3}$ $(0.017 \mathrm{~g}, 0.082 \mathrm{mmol})$ was then added after $4 \mathrm{~h}$ and the reaction mixture was stirred at room temperature overnight. The reaction mixture was diluted with satd. $\mathrm{NaHCO}_{3}$, extracted with EtOAc (2x), dried $\left(\mathrm{Na}_{2} \mathrm{SO}_{4}\right)$, filtered and concentrated. The residue was purified by chromatography on $\mathrm{SiO}_{2}(0-15 \%$ $\mathrm{MeOH} / \mathrm{CH}_{2} \mathrm{Cl}_{2}$ with $\left.0.1 \% \mathrm{Et}_{3} \mathrm{~N}\right)$ followed by filtration through basic alumina $\left(0-4 \% \mathrm{MeOH} / \mathrm{CH}_{2} \mathrm{Cl}_{2}\right)$ to give the product $(8,0.045 \mathrm{~g}, 45 \%)$ as a light yellow foamy solid. IR (neat) $3315,2931,2825,2140,2106,1599$, 1459, 1287, 1123, 773, $686 \mathrm{~cm}-1 ;{ }^{1} \mathrm{H}$ NMR (400 MHz, acetone-d 6 ) $\delta 11.21$ (bs, $1 \mathrm{H}$ ), 7.99 (t, J=0.8 Hz, 1 H), $7.55(\mathrm{dt}, \mathrm{J}=8.4,0.7 \mathrm{~Hz}, 1 \mathrm{H}), 7.46(\mathrm{t}, \mathrm{J}=1.5 \mathrm{~Hz}, 1 \mathrm{H}), 7.39(\mathrm{dd}, \mathrm{J}=8.4,1.6 \mathrm{~Hz}, 1 \mathrm{H}), 7.35-7.26(\mathrm{~m}, 3 \mathrm{H})$, 7.21-7.18 (m, $1 \mathrm{H}), 7.15(\mathrm{t}, \mathrm{J}=1.7 \mathrm{~Hz}, 1 \mathrm{H}), 7.01(\mathrm{dd}, \mathrm{J}=2.2,0.8 \mathrm{~Hz}, 1 \mathrm{H}), 6.96-6.92(\mathrm{~m}, 2 \mathrm{H}), 3.86(\mathrm{~s}, 2 \mathrm{H})$, 3.76 (dt, J=12.8, 3.4 Hz, 2 H), 2.84 (td, J=11.9, $2.1 \mathrm{~Hz}, 2 \mathrm{H}), 2.69$ (tt, J=10.0, 3.9 Hz, $1 \mathrm{H}$ ), 2.00 (dt, J=9.3, $3.6 \mathrm{~Hz}, 2 \mathrm{H}), 1.56-1.46(\mathrm{~m}, 2 \mathrm{H}) ;{ }^{13} \mathrm{C}$ NMR $(100 \mathrm{MHz} \text {, acetone-d })_{6} \delta 152.2,144.1,141.5,139.8,138.9$, 132.2, 129.6, 129.1, 125.3, 124.7, 124.2, 120.3, 118.4, 117.1, 115.99, 115.94, 112.8, 112.2, 102.6, 99.4, 54.0, 49.9, 47.7, 32.2; HRMS (ESI) m/z calcd. for $\mathrm{C}_{27} \mathrm{H}_{26} \mathrm{~N}_{7}(\mathrm{M}+\mathrm{H})$ 448.2244, found 448.2241.

2-(3-(4-((3-Azidobenzyl)amino)piperidin-1-yl)phenyl)-1H-indole-5-carbonitrile (11). A procedure adapted from Huryn, D.M.; Wipf, P.; LaPorte, M.G.; Colombo, R.; Kovaliov, M.; Lim, C.; Alverez, C.N.; Yue, Z.; Samankumara, L.P.; Chatterley, A.J.; Yan, Y.; Liang, M.; Green, N.J.; Moore, W.; Baldwin, E.; Arkin, M.R.; Neitz, J.; Bulfer, S.; Ang, K.-H.; Clifford, B. "Phenylindole derivatives as allosteric inhibitors of p97 ATPase and their preparation", WO2017/070320 was used. A solution of 1-(3-(1H-indol-2yl)phenyl)piperidin-4-one (9, 0.050 g, 0.172 mmol; Org. Biomol. Chem. 15, 40964114 (2017); ACS Med. Chem. Lett. 9, 1075-1081 (2018)) in 1,2-DCE (3 mL) was

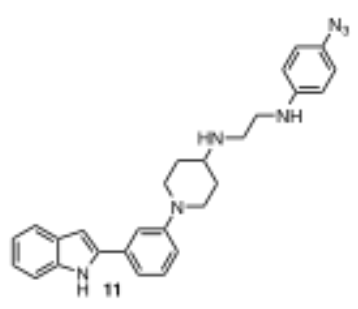
treated with N1-(4-azidophenyl)ethane-1,2-diamine (10, $0.046 \mathrm{~g}, 0.258 \mathrm{mmol}$; Bioconjugate Chem. 7, 343-348 (1996)) followed by $\mathrm{Ti}(\mathrm{OiPr})_{4}(0.058 \mathrm{~mL}, 0.19 \mathrm{mmol})$ at room temperature. After $30 \mathrm{~min}$, $\mathrm{NaBH}(\mathrm{OAc})_{3}(0.025 \mathrm{~g}, 0.12 \mathrm{mmol})$ was added. After $1 \mathrm{~h}$, additional $\mathrm{NaBH}(\mathrm{OAc})_{3}(0.025 \mathrm{~g}, 0.12 \mathrm{mmol})$ was added. Additional $\mathrm{NaBH}(\mathrm{OAC})_{3}(0.025 \mathrm{~g}, 0.12 \mathrm{mmol})$ was then added after $1 \mathrm{~h}$ and the reaction mixture was stirred at room temperature overnight. The reaction mixture was diluted with satd. $\mathrm{NaHCO}_{3}$, 
extracted with EtOAc $(2 \mathrm{x})$, dried $\left(\mathrm{Na}_{2} \mathrm{SO}_{4}\right)$, filtered and concentrated. The residue was purified by chromatography on $\mathrm{SiO}_{2}\left(0-10 \% \mathrm{MeOH} / \mathrm{CH}_{2} \mathrm{Cl}_{2}\right)$ followed by filtration through basic alumina $(0-10 \%$ $\mathrm{MeOH} / \mathrm{CH}_{2} \mathrm{Cl}_{2}$ ) to give the product $(\mathbf{1 1}, 0.035 \mathrm{~g}, 45 \%)$ as an off-white foamy solid. IR (neat) 3058, 3027, 2975, 2934, 2816, 2125, 1629, 1505, 1450, 1358, 1215, 1029, 982, 917, 839, 797, 734, $667 \mathrm{~cm}-1$; ${ }^{1} \mathrm{H}$ NMR (500 MHz, acetone- $\left.d_{6}\right) \delta 10.65$ (bs, $\left.1 \mathrm{H}\right), 7.56(\mathrm{~d}, \mathrm{~J}=8.0 \mathrm{~Hz} 1 \mathrm{H}), 7.46(\mathrm{~s}, 1 \mathrm{H}), 7.31-7.28(\mathrm{~m}, 2 \mathrm{H}), 7.10(\mathrm{t}$, $\mathrm{J}=7.5 \mathrm{~Hz}, 1 \mathrm{H}), 7.02(\mathrm{t}, \mathrm{J}=7.5 \mathrm{~Hz}, 1 \mathrm{H}), 6.96-6.88(\mathrm{~m}, 1 \mathrm{H}), 6.89-6.82(\mathrm{~m}, 3 \mathrm{H}), 6.71(\mathrm{~d}, \mathrm{~J}=8.6 \mathrm{~Hz}, 2 \mathrm{H}), 5.13$ (bs, $1 \mathrm{H}$ ), 3.78 (app d, J=12.8 Hz, $2 \mathrm{H}), 3.24-3.18(\mathrm{~m}, 2 \mathrm{H}), 2.95-2.83(\mathrm{~m}, 4 \mathrm{H}), 2.74-2.65(\mathrm{~m}, 1 \mathrm{H}), 2.04-$ $1.94(\mathrm{~m}, 2 \mathrm{H}), 1.54-1.43(\mathrm{~m}, 2 \mathrm{H}) ;{ }^{13} \mathrm{C}$ NMR $\left(125 \mathrm{MHz}, \mathrm{CDCl}_{3}\right): \delta 152.2,147.2,138.7,137.4,133.3,129.4$, 129.3, 127.4, 121.5, 120.1, 119.7, 119.5, 115.7, 115.2, 113.6, 112.6, 111.0, 110.9, 98.8, 54.5, 47.8, 45.4, 44.0, 32.4; HRMS (ESI) m/z calcd. for $\mathrm{C}_{27} \mathrm{H}_{30} \mathrm{~N}_{7}(\mathrm{M}+\mathrm{H})$ 452.2557, found 452.2554.

1,2-Distearoyl-sn-glycero-3-phosphoethanolamine-N-poly(ethylene glycol[5000])- $\mathrm{N}$-amidosuccinyldibenzocyclooctyne (DBCO-PEG5000-DSPE). DSPE-PEG5000- $\mathrm{NH}_{2}(50 \mathrm{mg}, 0.01 \mathrm{mmol})$ and NHS-DBCO (20.1 mg, $0.05 \mathrm{mmol}$ ) were dissolved in $2 \mathrm{~mL}$ of $\mathrm{CHCl}_{3}$ in a $10 \mathrm{~mL}$ round bottom flask with a stir bar. DIPEA (18 $\mu \mathrm{L}, 0.102 \mathrm{mmol}$ ) was added and the flask evacuated before backfilling with $\mathrm{N}_{2}$. The solution was stirred at $40{ }^{\circ} \mathrm{C}$ for $17 \mathrm{~h}$ and the reaction progress monitored by TLC. The volatiles were then removed under reduced pressure and residue purified by $\mathrm{SiO}_{2}$ column chromatography using a gradient mobile phase, starting with 95:5 DCM:MeOH and increasing in polarity to 80:20 DCM:MeOH, to yield DSPEPEG5000-DBCO as a solid white powder after solvent removal. Yield: 50\%. TLC: $\mathrm{Rf}=0.85$ (80:20 DCM:MeOH). ${ }^{1} \mathrm{H}$ NMR $\left(\mathrm{CDCl}_{3}\right): \delta 0.90(\mathrm{t}, 6 \mathrm{H}), 1.15-1.45(\mathrm{~m}, 44 \mathrm{H}), 2.25(\mathrm{~s}, 4 \mathrm{H}), 2.28-2.32(\mathrm{~m}, 2 \mathrm{H}), 2.48-$ $2.53(\mathrm{~m}, 2 \mathrm{H}), 2.75-2.78(\mathrm{~m}, 4 \mathrm{H}), 2.48-3.7(\mathrm{~m}, 180 \mathrm{H}), 3.55-3.60(\mathrm{~m}, 400 \mathrm{H}) 7.40-7.80(\mathrm{~m}, 8 \mathrm{H})$.

\section{Expression and purification of human p97}

A plasmid containing the gene for human p97 (kindly given as a gift from Tsui-Fen Chou, UCLA) was transformed into $E$. coli Rosetta ${ }^{\mathrm{TM}}$ (DE3) (MilliporeSigma). A single colony was inoculated into $50 \mathrm{~mL}$ LB media containing $100 \mathrm{mg} / \mathrm{L}$ ampicillin and $15 \mathrm{mg} / \mathrm{L}$ chloramphenicol. Inoculation of a $50 \mathrm{~mL}$ starter culture into $1 \mathrm{~L} \mathrm{LB}$ media containing $100 \mathrm{mg} / \mathrm{L}$ ampicillin was incubated with shaking at $37{ }^{\circ} \mathrm{C}$ until the culture produced an OD600 of 0.8 . The culture was then cooled to $20^{\circ} \mathrm{C}$, induced with $0.4 \mathrm{mM} \mathrm{IPTG}$, and the cells were harvested after $15 \mathrm{~h}$. Cell pellets ( $\sim 3 \mathrm{~g})$ from $1 \mathrm{~L}$ of culture media were suspended in 30 $\mathrm{mL}$ of lysis buffer (100 mM Tris, pH 7.4, $500 \mathrm{mM} \mathrm{KCl}, 5 \mathrm{mM} \mathrm{MgCl}$, $20 \mathrm{mM}$ imidazole, 5\% glycerol, $2 \mathrm{mM}$ 2-mercaptoethanol, Roche protease inhibitor tablet) and lysed by six $30 \mathrm{~s}$ pulses of probe sonication (pellet held in ice bath), separated by 2 min intervals. The lysate was then centrifuged at 20,000 $\mathrm{g}$ for $45 \mathrm{~min}$ at $4{ }^{\circ} \mathrm{C}$ and the resulting supernatant was loaded onto Ni-NTA super flow columns (Qiagen Super Flow Ni-NTA column) ( $5 \mathrm{~mL}$ suspension) pre-equilibrated with wash buffer (50 mM HEPES, pH 7.4, 150 $\mathrm{mM} \mathrm{KCl}, 5 \mathrm{mM} \mathrm{MgCl} 2,20 \mathrm{mM}$ imidazole) before incubating at $4{ }^{\circ} \mathrm{C}$ with rotation for $30 \mathrm{~min}$. The column was then flushed with wash buffer $(100 \mathrm{~mL})$ and His6-tagged $\mathrm{p} 97$ was eluted by stepwise application of $10 \mathrm{~mL}$ of imidazole elution buffer $(50,100,150,200$, and $250 \mathrm{mM}$ imidazole in wash buffer step wise). Fractions from the 150, 200, and $250 \mathrm{mM}$ imidazole steps were combined and concentrated with Amicon Ultra-15 centrifugal filter units (NMWL $=100 \mathrm{kDa}$ ). The retentate $(1 \mathrm{~mL}$ of $20 \mathrm{mg} / \mathrm{mL}$ ) was then fractionated using a SuperdexTM 200 10/300 GL column (GE Healthcare Life Sciences) in GF buffer (20 mM HEPES, pH 7.4, $250 \mathrm{mM} \mathrm{KCl}, 1 \mathrm{mM} \mathrm{MgCl}_{2}$ ) at a flow rate of $0.5 \mathrm{~mL} / \mathrm{min}$, and fractions corresponding to 540 kDa were collected. Freshly purified p97 concentration was measured using Bradford assays. 


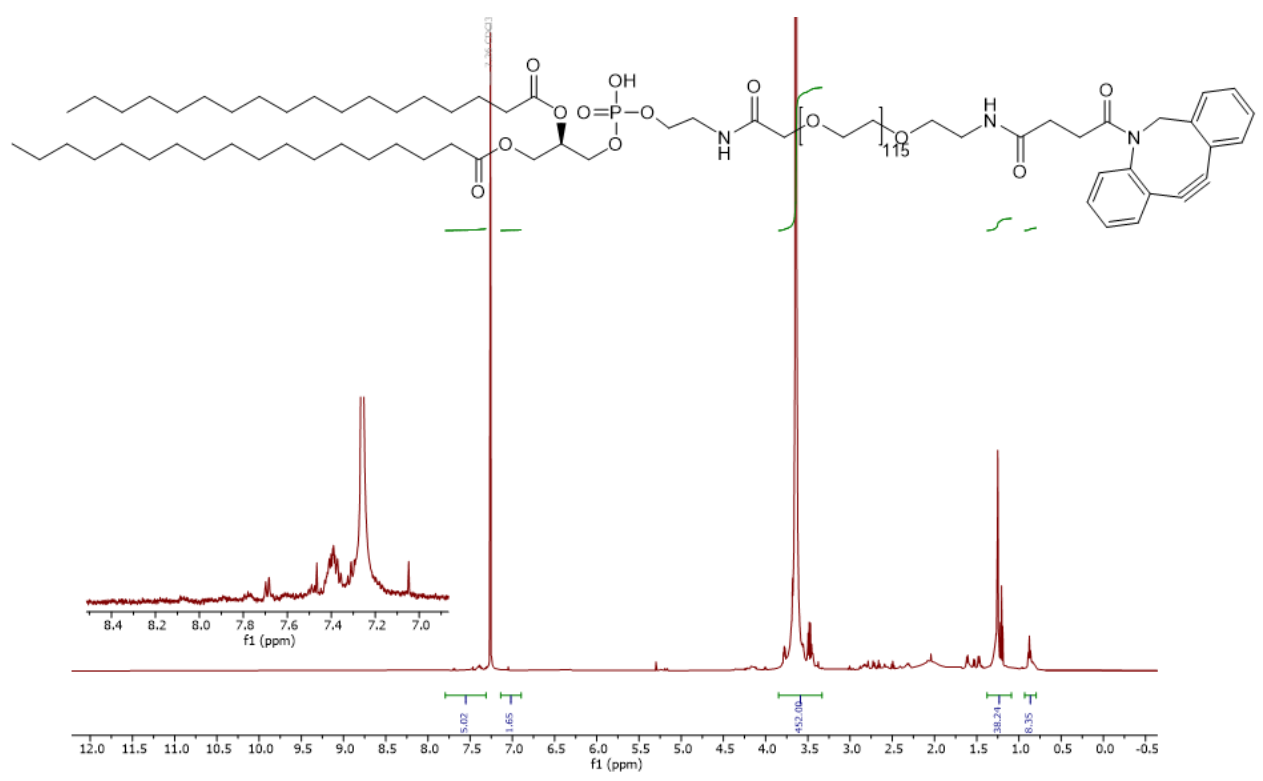

Figure S1. 1H-NMR of DBCO-PEG5000-DSPE.

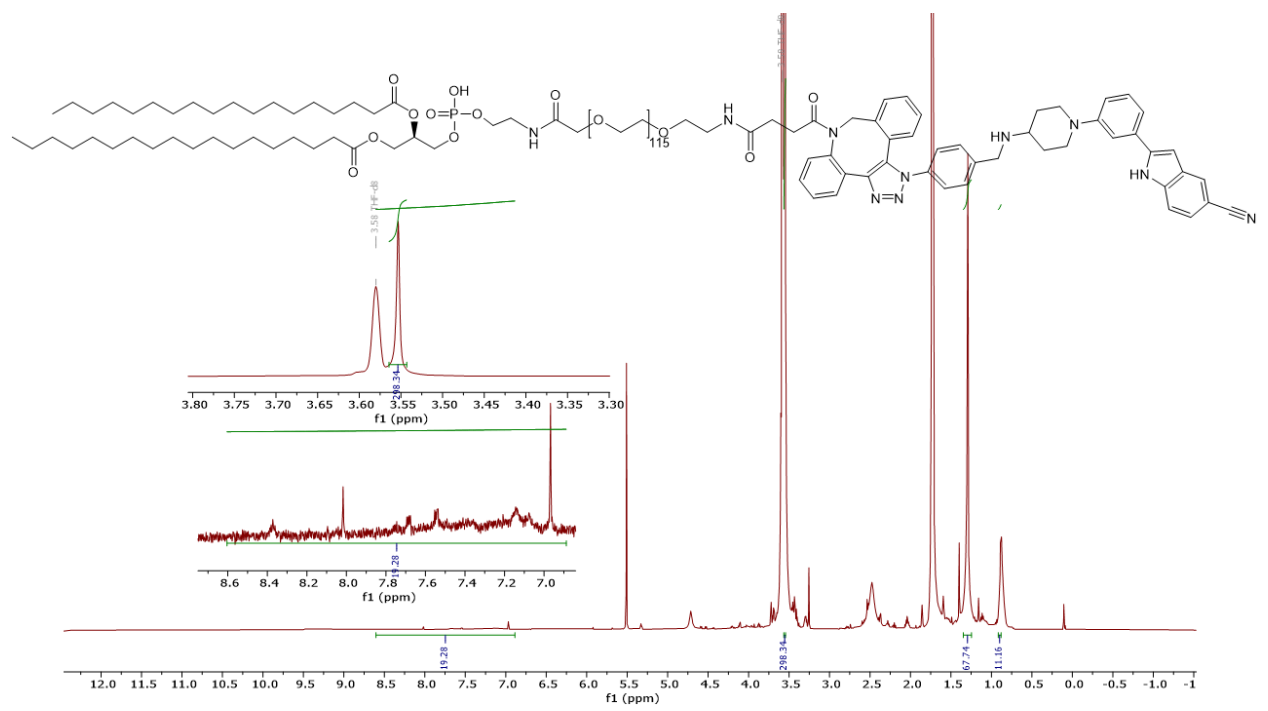

Figure S2. 1H-NMR of 1 (Cyanoindole ligand-PEG5000-DSPE conjugate). 


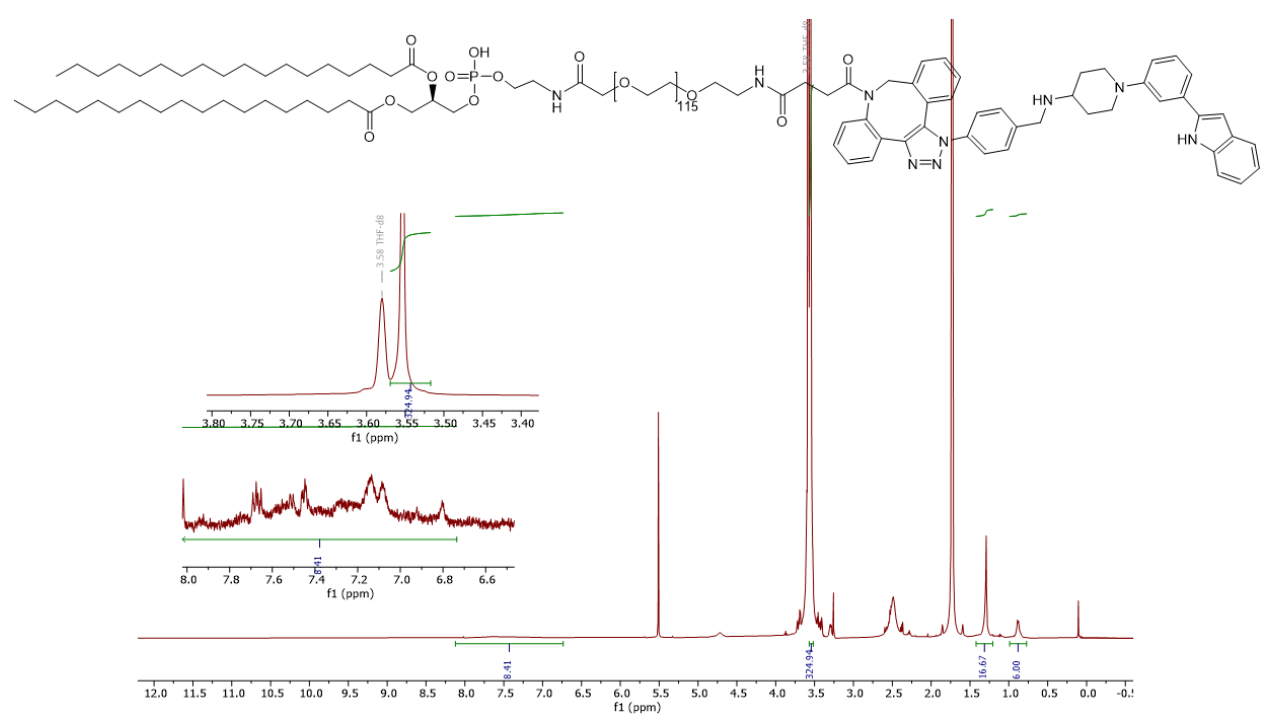

Figure S3. 1H-NMR of 3 (Indole ligand-PEG5000-DSPE conjugate).

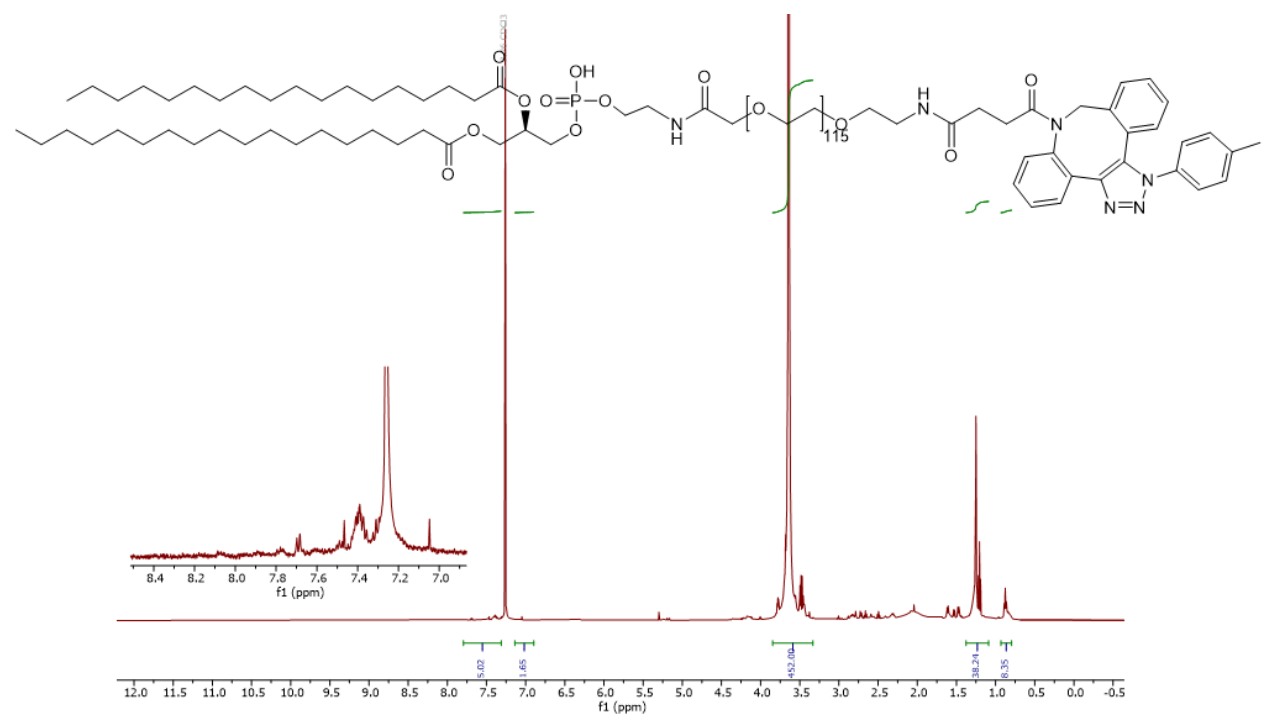

Figure S4. 1H-NMR of 4 (4-Tolyl triazole-PEG5000-DSPE conjugate). 


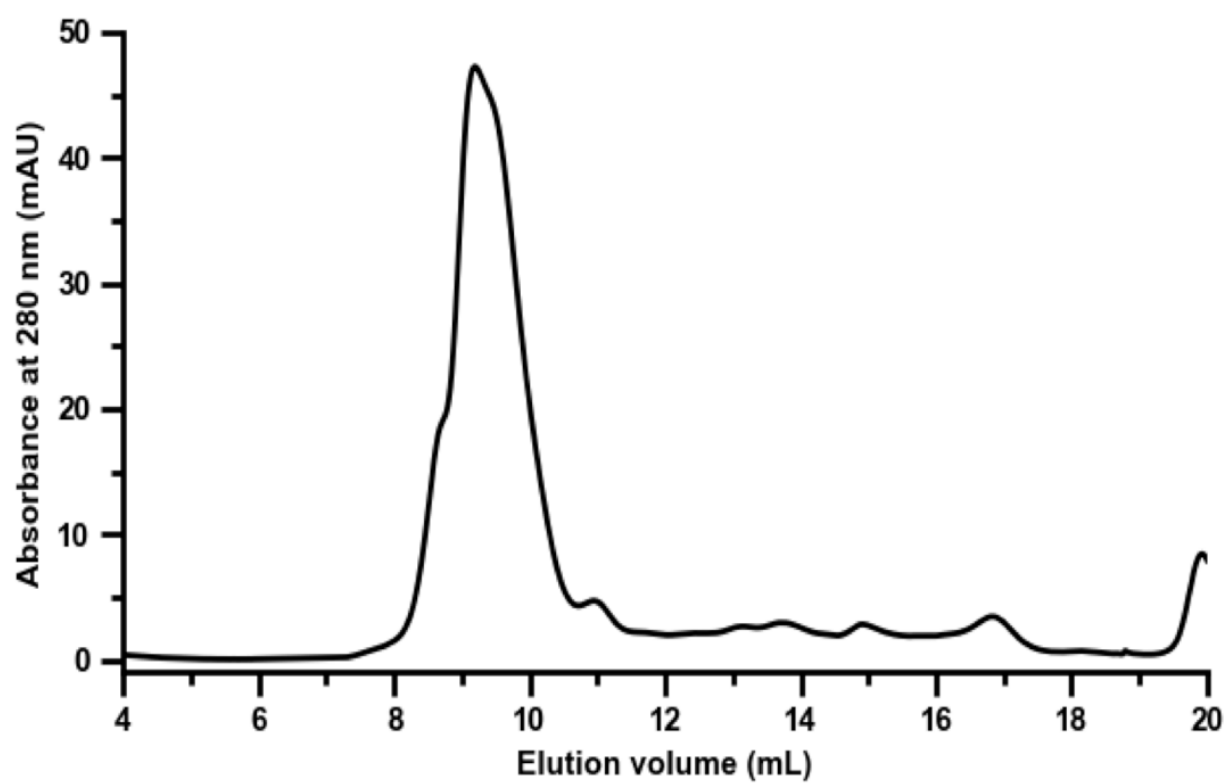

Figure S5. SEC chromatogram of isolated His6-tagged p97. The elution was performed using an ÄKTA Pure 25 M (GE Healthcare Life Sciences) with HEPES buffer, flow rate $=0.2 \mathrm{~mL} / \mathrm{min}$, and a SuperdexTM $20010 / 300 \mathrm{GL}$ column. The injected volume was $70 \mu \mathrm{L}$ of a $6.1 \mathrm{mg} / \mathrm{mL} \mathrm{p} 97 \mathrm{solution}$. Fractions were collected at $300 \mu \mathrm{L}$ increments. The p97 elutes after $40 \mathrm{~min}$ with the main peak appearing around $46 \mathrm{~min}$ and a shoulder at $43 \mathrm{~min}$.
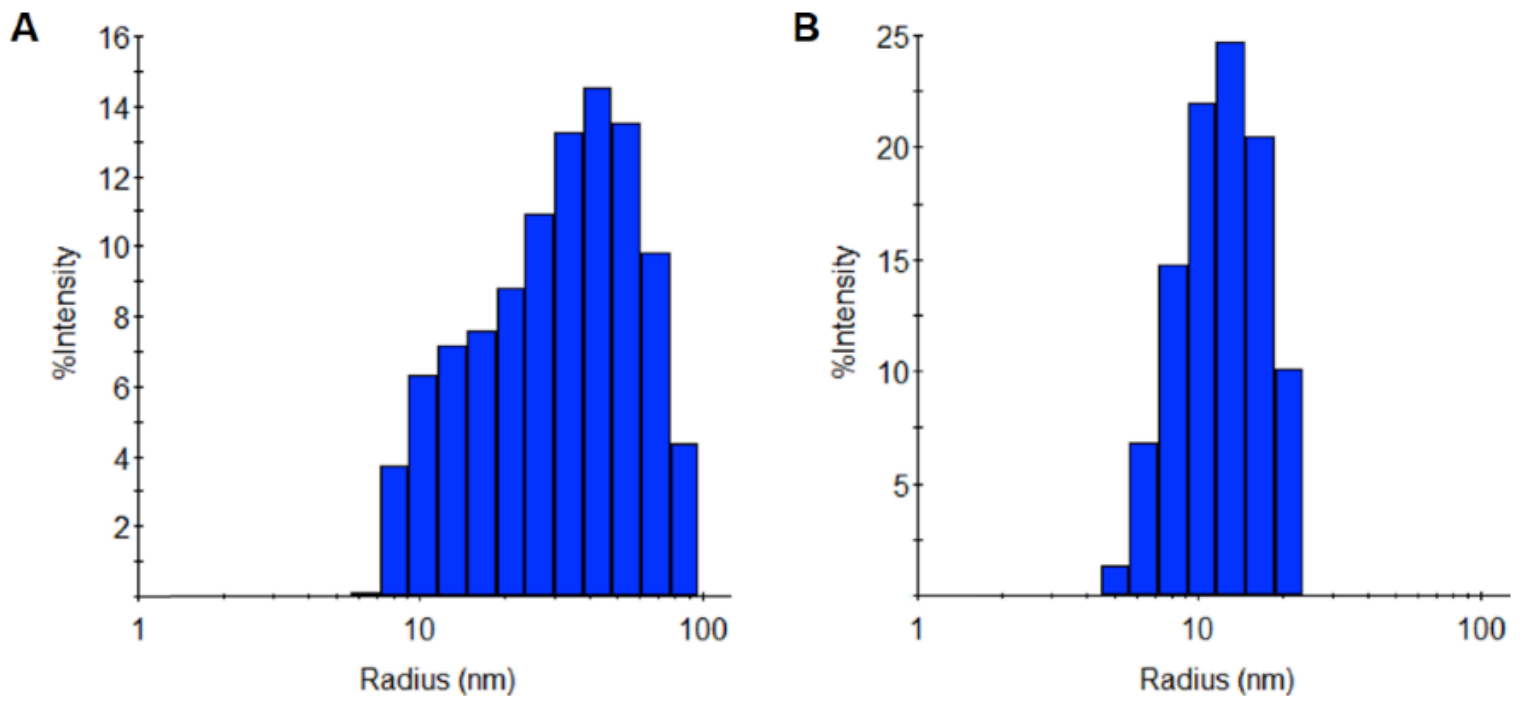

Figure S6. Dynamic light scattering analysis of p97 fractions from SEC. Intensity distribution of a fraction collected from the leading edge of the SEC curve (A) reveals the presence of a larger particle $(40 \mathrm{~nm})$ that is more than double the diameter of a sample analyzed from the main chromatographic peak (B) $(13 \mathrm{~nm})$. Data were collected using a DynoPro ${ }^{\circledR}$ PlateReader II. 


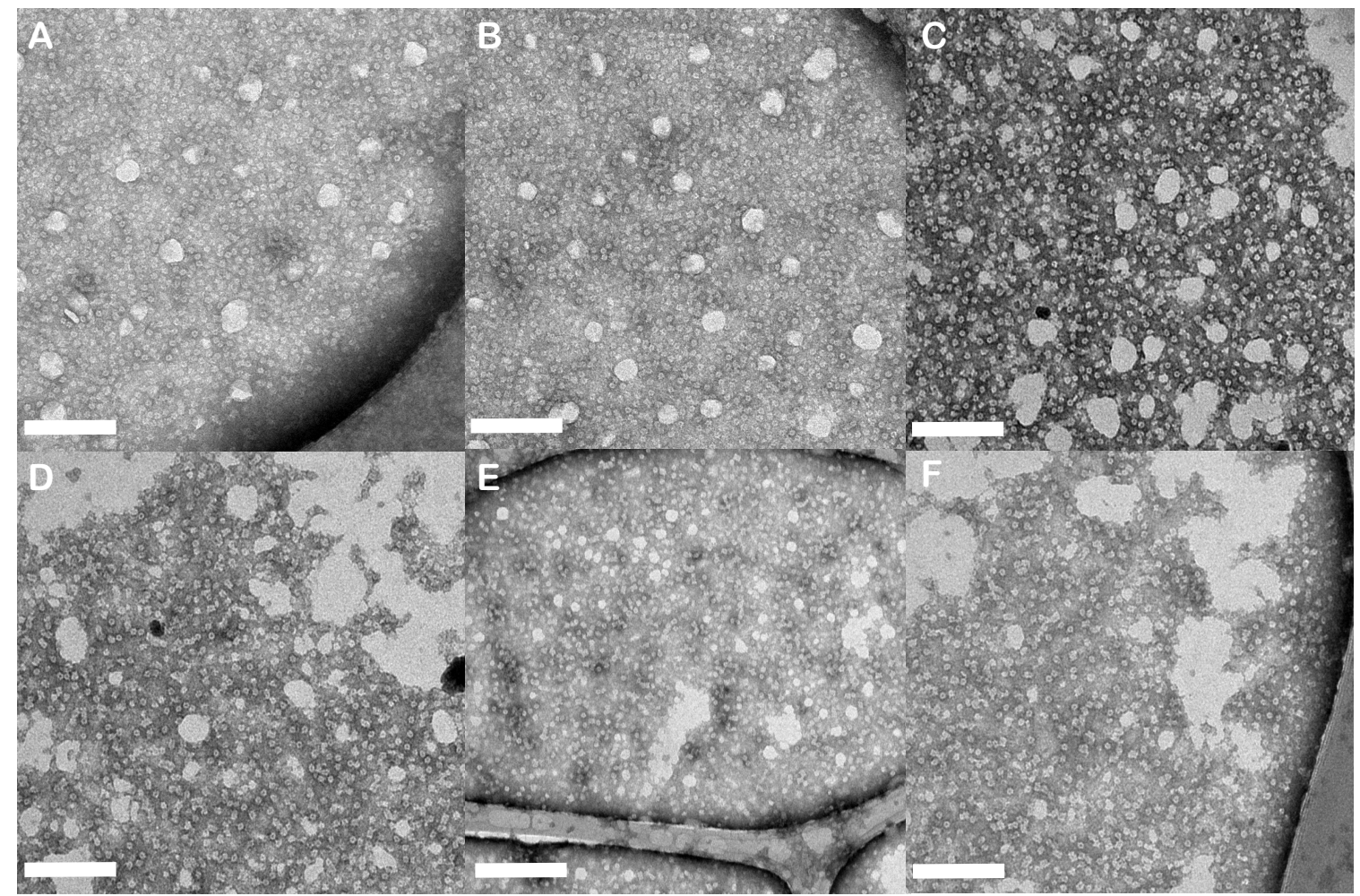

Figure S7. Negative stain TEM micrographs of p97 captured by 1:99 1:mPEG750-DSPE coated grids using different concentrations of p97. (A) 200 $\mu \mathrm{g} / \mathrm{mL}$; (B) $100 \mu \mathrm{g} / \mathrm{mL}$; (C) $50 \mu \mathrm{g} / \mathrm{mL}$; (D) $40 \mu \mathrm{g} / \mathrm{mL}$; (E) $30 \mu \mathrm{g} / \mathrm{mL}$; (F) $15 \mu \mathrm{g} / \mathrm{mL}$. Scale bar = $100 \mathrm{~nm}$.

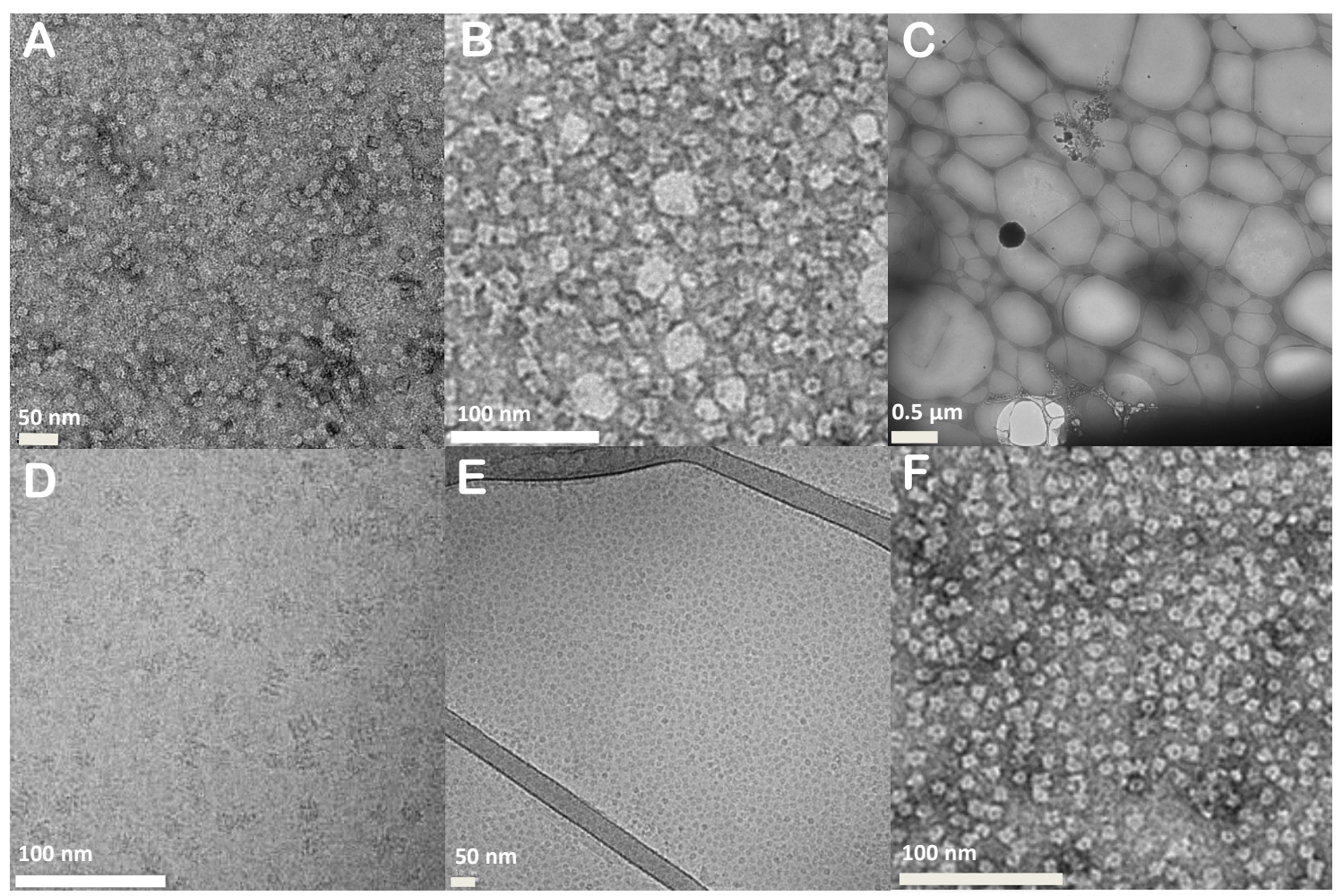

Figure S8. Negative-stain and cryo-EM micrographs of p97 captured with grids coated with different 1:mPEG750-DSPE ratios using $15 \mu \mathrm{g} / \mathrm{mL}$ solutions. All grids were prepared and imaged as described in Materials and Methods. (A) Negative stain TEM of p97 captured with 1:99 1:mPEG750-DSPE coated grids. (B) Negative stain TEM of p97 captured with 3:97 1:mPEG750-DSPE coated grids. (C) Low magnification cryo-EM image of p97 captured with 1:99 1:mPEG750- 
DSPE coated grids. (D) High magnification cryo-EM image of p97 captured with 1:99 1:mPEG750-DSPE coated grids. (E) Low magnification cryo-EM image of p97 captured with 3:97 1:mPEG750-DSPE coated grids. (F) Negative stain TEM of p97 captured with 3:97 1:mPEG750-DSPE coated grids. 

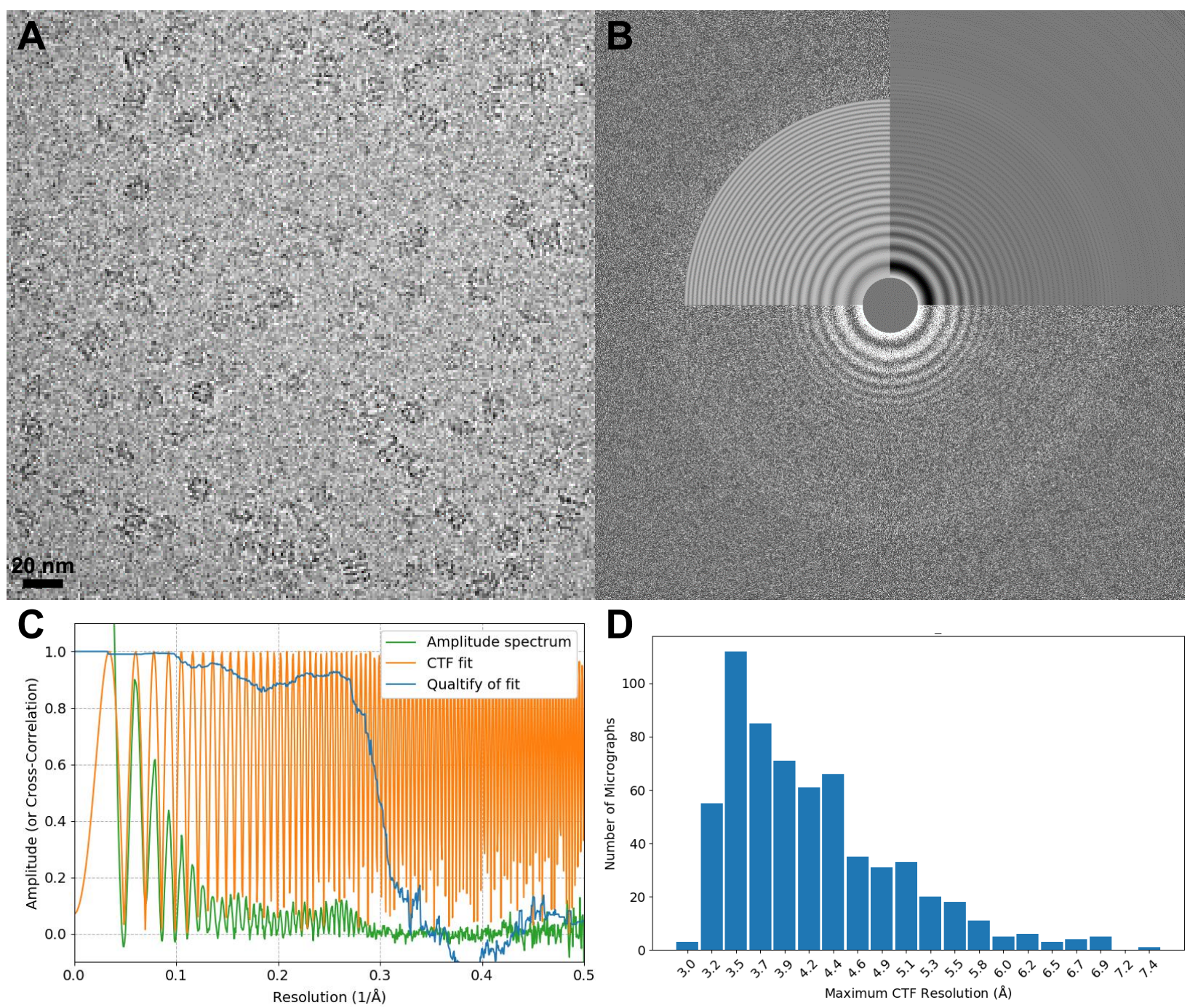

Figure 59. Quality of acquired data. (A) Sample micrograph from Titan Krios dataset. (B) 2D Thon rings of micrograph in (A). (C) 1D power spectra of (B) showing quality of fit. (D) Histogram of maximum CTF resolution of entire dataset. 
A

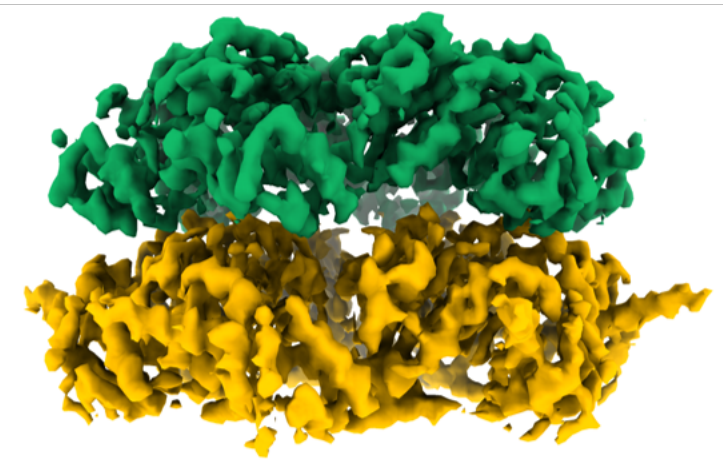

C

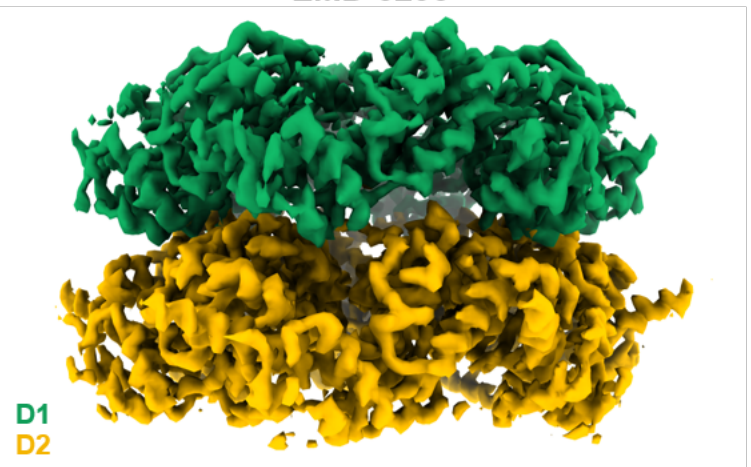

B One Half of Double-hexamer

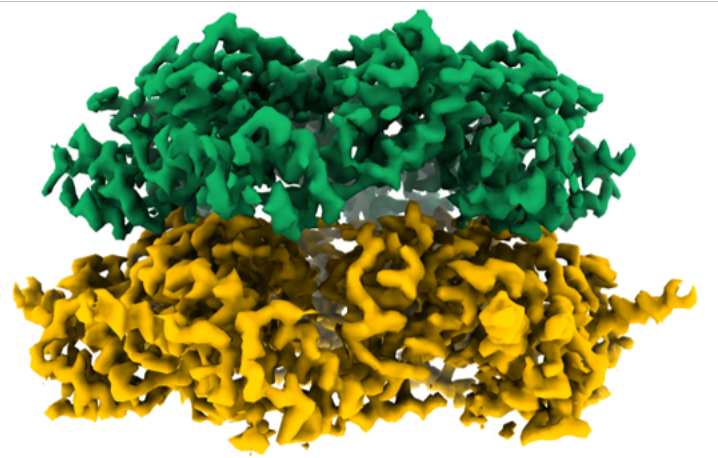

D

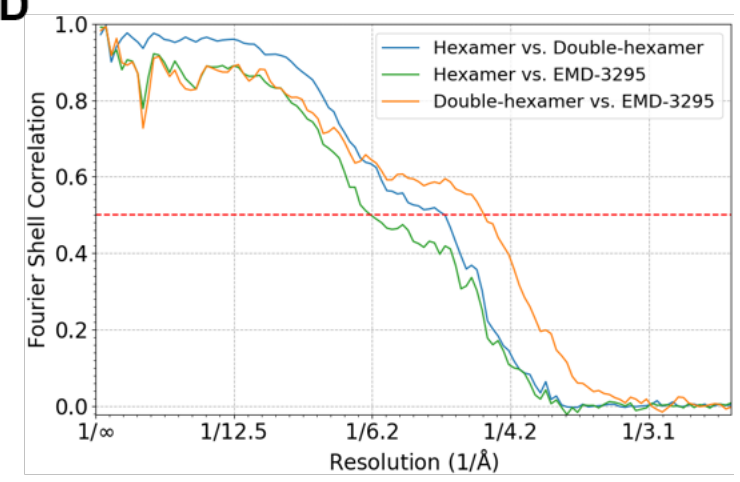

Figure S10. Comparison of our cryo-EM maps vs. the reference p97 map18. The densities observed in the (A) C6 and (B) D6 states compared similarly to the (C) 2.3 Å cryo-EM structure (EMDB-3295). Density maps in (A-C) were sharpened with -215.79, -76.27 , and +56.74 Å2 B-factors, respectively. (D) FSC plot of our maps versus each other and versus EMD-3295. Panels (A-C) were generated in ChimeraX (TD Goddard et al. Protein Sci. 2018 27, 14-25). 